\title{
A suplementação de glutamina é benéfica em crianças com doenças graves?
}

\author{
Glutamine supplementation: Is it beneficial to \\ critically ill children?
}

Stefânia Lucizani PACÍFICO'1

Heitor Pons LEITE'

Werther Brunow de CARVALHO²

\section{R E S U M O}

Embora haja vários estudos clínicos randômicos avaliando os benefícios da suplementação de glutamina em pacientes adultos, há pouca informação em pacientes pediátricos, particularmente naqueles com doenças graves. O objetivo deste estudo foi avaliar criticamente os estudos sobre suplementação de glutamina, para verificar os possíveis benefícios clínicos desta suplementação em crianças gravemente doentes. Levou-se a cabo uma pesquisa dos estudos publicados entre 1992 e 2003, do tipo prospectivo, randômico e controlado, conduzidos em pacientes pediátricos gravemente doentes, divididos em dois grupos: o dos que receberam suplementos de glutamina por via enteral ou parenteral, e o dos que receberam placebo. As variáveis de desfecho foram: tempo para atingir oferta plena de dieta enteral, tempo de hospitalização, complicações e mortalidade. Foram utilizadas as bases de dados Medline, LILACS e Cochrane, com as palavras-chave: glutamine, critically ill, trauma, sepsis, burned, injured, bone marrow transplantation, intensive care, mechanical ventilation. Dos 33 estudos inicialmente identificados, 7 preencheram os critérios de inclusão. A análise dos resultados mostrou que nos pacientes suplementados com glutamina, houve melhor aceitação por via enteral e redução do tempo de nutrição parenteral em recém-nascidos, não havendo efeito sobre o tempo de internação, custos hospitalares e mortalidade. Os estudos controlados na faixa etária pediátrica, não permitem que se adote o uso rotineiro da glutamina em crianças gravemente enfermas. O número reduzido de pacientes e a heterogeneidade quanto ao grupo etário, doenças e via de administração são fatores que limitam a interpretação dos resultados.

Termos de indexação: criança, glutamina, nutrição enteral, nutrição parenteral, suplementação, terapia intensiva.

\footnotetext{
1 Unidade de Terapia Intensiva, Hospital do Servidor Público Estadual. Rua Pedro de Toledo, 1800, 04038-000, São Paulo, SP, Brasil. Correspondência para/Correspondece to: S.L. PACÍFICO.E-mail: <stefanianeo@uol.com.br>.

2 Departamento de Pediatria, Unidade de Terapia Intensiva, Unidade Federal de São Paulo, Escola Paulista de Medicina. São Paulo, SP, Brasil.
} 
96 S.L. PACÍFICO et al.

\section{A B S T R A C T}

Although there have been several randomized trials, conducted with critically ill adults, reporting the benefits of glutamine supplementation, there is scarce information on the role of glutamine in the treatment outcome of critically ill children. Our objective was to evaluate existing studies on the effect of glutamine supplementation, aiming at verifying the clinical benefits of such supplementation for critically ill children. A computerized search was conducted to select prospective, randomized, clinical trials of critically ill children, published between 1992 and 2003. Studies were included if, besides presenting the characteristics already mentioned, they evaluated the effects of parenteral and the enteral glutamine supplementing vs. those of standard care. The variables on clinical outcomes were: time to achieve full enteral nutrition, hospital length of stay, complication rates, and mortality. Three bibliographic databases were searched: Medline, LILACS and Cochrane Library. Search terms included: glutamine, critically ill, trauma, sepsis, burned, injured, bone marrow transplantation, intensive care, and mechanic ventilation. Initial screening resulted in 33 original articles; of these potentially eligible articles, only 7 fulfilled the inclusion criteria. Length of hospital stay, mortality rate, and hospital costs were not different for patients receiving glutamine-supplemented nutrition, when compared to those receiving non-supplemented diet. Glutamine was associated with reduction in the time necessary to achieve full enteral feeding and parenteral nutrition. There is no evidence to support the routine use of either parenteral or enteral glutamine supplement when treating critically ill children. The small number of patients, heterogeneity with respect to the studied groups, routes of administration, and confounding effects of concomitant diseases, all limit the inferences we can make from the results.

Indexing terms: child, glutamine, enteral nutrition, parenteral nutrition, suplementary feeding, intensive care.

\section{N T R O D U Ç Ã O}

A glutamina é o aminoácido livre mais abundante no músculo e no plasma humano, sendo também encontrada em concentrações relativamente elevadas em muitos tecidos. É um aminoácido essencial para o crescimento e a diferenciação celular, transporte de cadeia carbônica entre os órgãos e fornecimento de energia para células de rápida proliferação, como os enterócitos e as células do sistema imune. Além disso, atua como precursora da ureagênese e neoglicogênese hepática e de mediadores como o GABA e o glutamato'.

Apesar da grande reserva muscular de glutamina, os estoques endógenos podem reduzir-se, em adultos e crianças, durante insultos catabólicos tais como grandes cirurgias, queimaduras extensas, septicemia e inflamação. A demanda metabólica excede a sua capacidade de síntese, sendo necessária a suplementação pela dieta. Devido a esta particularidade, a glutamina foi recentemente classificada como um aminoácido condicionalmente essencial ${ }^{2}$.

Nestas circunstâncias de hiper-metabolismo, há aumento dos hormônios catabólicos ( $\mathrm{CRH}$, ACTH, glicocorticóides, catecolaminas), que exacerbam a proteólise e a neoglicogênese ${ }^{3}$, excedendo a capacidade de síntese de glutamina no músculo esquelético e ocasionando a diminuição das reservas e da concentração plasmática. Além disso, acredita-se que, recém-nascidos prematuros com baixo peso ao nascimento, por apresentarem reservas energéticas reduzidas e pouca musculatura esquelética, sejam mais susceptíveis a estados de deficiência de glutamina.

As repercussões no organismo da depleção de glutamina vêm sendo objeto de muitos estudos, sobretudo no que se refere ao sistema imune e à susceptibilidade às infecções, já que sua metabolização pelas células imunológicas fornece substratos fundamentais para a formação 
de membranas celulares durante a atividade das células fagocitárias, síntese de citocinas (fator de necrose tumoral e interleucinas), e ânion superóxido ${ }^{4}$. Assim, a fagocitose, a degranulação e a produção de citocinas por neutrófilos e macrófagos, estão intimamente relacionadas à disponibilidade de glutamina, de tal forma que situações de estresse podem induzir a imunossupressão.

Visto que a glutamina é utilizada como fonte energética preferencial por enterócitos, a sua depleção exerce impacto negativo sobre a celularidade da mucosa intestinal. Isto contribui para alterações na função de barreira do epitélio digestivo, as quais, aliadas a outras situações que debilitam a mucosa, como a má perfusão e as alterações hormonais - acabam por predispor à translocação bacteriana e à conseqüente sepse ${ }^{5,6}$.

Possivelmente, a deficiência de glutamina pode limitar, tanto a produção de proteínas na resposta inflamatória, como a síntese da glutationa, comprometendo as defesas antioxidantes do organismo.

Os potenciais efeitos benéficos da suplementação da glutamina podem ser ${ }^{7}$ :

- Aumentar a síntese de glutationa, potencializando as defesas antioxidantes.

- Manter a integridade da mucosa intestinal (fonte energética para enterócitos), evitando a translocação bacteriana.

- Aumentar a síntese de proteínas da resposta inflamatória, atenuando o processo inflamatório.

- Preservar a função imune, servindo de fonte energética para linfócitos e precursores de citocinas.

Nos últimos anos, o uso da glutamina mostrou-se efetivo em pacientes adultos criticamente enfermos. Conforme dados da metanálise, realizada por Novak et al., em 2002', a suplementação deste aminoácido promoveu redução das infecções e do tempo de internação no grupo de pacientes cirúrgicos, sendo verificada diminuição da mortalidade em pacientes críticos.
Os resultados mais expressivos e animadores foram obtidos com altas doses do aminoácido endovenoso. Todavia há uma lacuna de informações, no que concerne à faixa etária pediátrica, com exceção de uns poucos estudos abordando pacientes pediátricos em Unidade de Terapia Intensiva (UTI), que receberam suporte nutricional com glutamina. Em vista dos potenciais benefícios deste aminoácido, torna-se pertinente e relevante o interesse pelos efeitos da suplementação da glutamina em crianças com doenças graves.

Esta revisão da literatura procura avaliar os efeitos benéficos, clínicos e econômicos, da suplementação enteral e parenteral da glutamina em pacientes pediátricos gravemente doentes.

Para os critérios de revisão foram selecionados estudos prospectivos controlados e randômicos, sobre o papel da suplementação de glutamina em crianças e adolescentes com idade entre zero e 18 anos de ambos os sexos criticamente enfermos utilizando suplementação via enteral e parenteral, considerando a dosagem e o tempo de uso. As variáveis estudadas foram tempo de nutrição parenteral, tolerância à dieta enteral, infecções nosocomiais, mortalidade, tempo de internação e custo hospitalar. Revisaram-se e selecionaram-se os estudos publicados entre 1992 e 2003, utilizando as bases de dados Medline, LILACS e Cochrane. Os termos de indexação (indexing terms) utilizados para pesquisa foram: glutamine; critically ill; trauma; sepsis; burned; injured; bone marrow transplant; intensive care; mechanical ventilation. Foram encontrados 35 artigos e destes, apenas 79-15 preencheram os critérios de seleção. A síntese destes estudos estão apresentados nos Quadros $1,2,3,4,5,6$ e 7 .

\section{RESULTADOSE DISCUSSÃO}

Muitas dessas investigações sobre o uso clínico da glutamina mostraram benefícios clínicos e metabólicos que incluem a redução do tempo de hospitalização e a diminuição da taxa de infecção ${ }^{16-18}$. 
Quadro 1. Efeitos da suplementação enteral de glutamina sobre a morbidade de recém-nascidos de baixo peso.

\begin{tabular}{|c|c|c|c|}
\hline Autor & Participantes & Intervenção & Resultados \\
\hline \multirow[t]{4}{*}{ Neu et al. ${ }^{13}$} & Selecionados 68 RN & $\begin{array}{l}\text { RN divididos em } 2 \text { grupos: } 35 \text { trata- } \\
\text { dos e } 33 \text { controles. }\end{array}$ & $\begin{array}{l}\text { Grupo suplementado com } \\
\text { glutamina: }\end{array}$ \\
\hline & $\begin{array}{l}\text { Inclusão: RN com IG } 24 \text { a } 32 \text { semanas } \\
\text { e peso de nascimento entre } 500 \text { e } \\
1250 \mathrm{~g}\end{array}$ & $\begin{array}{l}\text { Glutamina: acrescentada na fórmula } \\
\left.\text { láctea } 0,08 \mathrm{~g} / \mathrm{kg} \text { no início ( } 3^{\circ} \mathrm{dia}\right) \text {, sen- } \\
\text { do aumentado } 0,31 \mathrm{~g} / \mathrm{kg} \text { por dia até }\end{array}$ & $\begin{array}{l}\text { - Maior tolerância à dieta enteral e } \\
\text { - Renor tempo de NP. }\end{array}$ \\
\hline & $\begin{array}{l}\text { Exclusão: RN com anomalia congênita } \\
\text { do TGl; enterocolite necrosante; } \\
\text { grandes cirurgias; aleitamento materno } \\
\text { antes do início do estudo; paciente } \\
\text { com malformação incompatível com a } \\
\text { vida. }\end{array}$ & $\begin{array}{l}13^{\circ} \text { dia. A glutamina foi suspensa no } \\
30^{\circ} \text { dia. } \\
\text { Progressão lenta enteral até atingir } \\
\text { nutrição plena em torno de } 150 \mathrm{~mL} \\
\mathrm{~kg} / \mathrm{d} \text {. } \\
\text { Suspensa a NP se infusão inferior a } \\
1,5 \mathrm{~mL} / \text { hora. }\end{array}$ & $\begin{array}{l}\text { - Tendência a menor tempo de } \\
\text { VPM. Aumentou incidência de } \\
\text { apnéia. } \\
\text { Não houve diferença significante } \\
\text { nas variáveis: Peso, PC, tempo de } \\
\text { internação, Apgar, IG, sexo, uso de } \\
\text { corticóide e surfactante. }\end{array}$ \\
\hline & $\begin{array}{l}\text { Exclusão durante estudo: } \\
\text { Sepse com interrupção da dieta; cirur- } \\
\text { gia e óbito. } \\
\text { Período: Março } 1992 \text { a julho } 1994 .\end{array}$ & $\begin{array}{l}\text { Controles: eletrólitos, função renal, } \\
\text { gasometria, dosagem de AA e amônia } \\
\text { Todos os RN foram avaliados nos pri- } \\
\text { meiros } 30 \text { dias de vida. }\end{array}$ & $\begin{array}{l}\text { Não houve diferença: nível sérico } \\
\text { de amônia, nitrogênio, glutamato, } \\
\text { glutamina, transaminases. }\end{array}$ \\
\hline
\end{tabular}

$\mathrm{IG}=$ idade gestacional; $\mathrm{AA}=$ aminoácidos; $\mathrm{NP}=$ nutrição parenteral; $\mathrm{PC}=$ perímetro cefálico; $\mathrm{RN}=$ recém-nascido; $\mathrm{VPM}=$ ventilação pulmonar mecânica; TGI= trato gastrintestinal.

Quadro 2. Efeitos da suplementação parenteral da glutamina em recém-nascidos prematuros.

\begin{tabular}{|c|c|c|c|}
\hline Autor & Participantes & Intervenção & Resultados \\
\hline Lacey et al. ${ }^{12}$ & $\begin{array}{l}\text { Participantes: } 78 \text { RN } \\
\text { Inclusão: RN }<4 \text { dias de vida, } \\
<3 \text { dias NP, alto risco para ECN, P } \\
<1500 \mathrm{~g}, \text { Ig<32sem, } \\
\text { Apgar } 5^{\circ}<6, \mathrm{PCA} \text {, convulsão, uso de } \\
\text { cateter umbilical e necessidade de } \\
\text { VPM. } \\
\text { Exclusão: lesão grave SNC, erro inato } \\
\text { do metabolismo, insuficiência renal e } \\
\text { hepática. } \\
\text { Exclusão durante estudo: } \\
\text { Glutamina <7dias, }<1,5 \mathrm{~g} \text { de AA/kg, } \\
\text { sepse com interrupção da dieta, cirur- } \\
\text { gia e óbito. } \\
\text { Período: Maio } 1990 \text { a Agosto } 1992 \\
\text { RN semelhantes quanto ao peso, IG, } \\
\text { sexo, Apgar, uso de corticosteróide e } \\
\text { surfactante. }\end{array}$ & $\begin{array}{l}\text { Foram divididos dois grupos: com e } \\
\text { sem suplementação de glutamina } \\
\text { Glutamina: acrescentada na NP nas } \\
\text { concentrações de } 15 \%, 20 \% \text { e } 25 \% \\
\text { do peso por volume da mistura de } \\
\text { aminoácidos. Sendo iniciada no } 4^{\circ} \text { dia } \\
\text { de vida e mantida pelo menos por } 7 \\
\text { dias. } \\
\text { Iniciado dieta enteral quando RN } \\
\text { estável e sem cateter umbilical. } \\
\text { Progressão lenta enteral se não } \\
\text { houvesse resíduos, distensão, sangue } \\
\text { nas fezes, sepse. } \\
\text { Suspensa a NP se infusão } \\
<1,5 m L / h o r a . \\
\text { Controles: eletrólitos, gasometria, do- } \\
\text { sagem de AA e amônia. }\end{array}$ & $\begin{array}{l}\text { - Glutamina: segura para uso em } \\
\text { - NNão houve diferença entre os } 2 \\
\text { grupos quanto à duração da NP, } \\
\text { ao tempo para atingir nutrição } \\
\text { enteral plena, à incidência de cul- } \\
\text { turas positivas, ao ganho de peso } \\
\text { e duração de internação. } \\
\text { - RN com peso<800g suplemen- } \\
\text { tados com glutamina, tiveram } \\
\text { menor tempo de NP e maior acei- } \\
\text { tação via enteral. } \\
\text { - Não houve diferença significante } \\
\text { no uso de hemoderivados e na } \\
\text { contagem de leucócitos. } \\
\text { - Houve tendência no grupo } \\
\text { suplementado a apresentar nível } \\
\text { sérico mais elevado de amônia, } \\
\text { uréia, glutamato e glutamina. }\end{array}$ \\
\hline
\end{tabular}

$\mathrm{RN}=$ recém-nascido; $\mathrm{NP}=$ nutrição parenteral; $\mathrm{ECN}=$ enterocolite necrosante; $\mathrm{PCA}=$ persistência do canal arterial; Ig=idade gestacional; $\mathrm{AA}=$ aminoácido; $\mathrm{SNC}=$ sistema nervoso central. 
Quadro 3. Efeitos da suplementação de glutamina enteral sobre os custos hospitalares.

\begin{tabular}{|c|c|c|c|}
\hline Autor & Participantes & Intervenção & Resultados \\
\hline Dallas et al. ${ }^{11}$ & $\begin{array}{l}\text { Foram selecionados } 68 \text { pacientes se- } \\
\text { melhantes quanto ao peso, sexo, lg, } \\
\text { Apgar, uso de corticosteróide e } \\
\text { surfactante. } \\
\text { Inclusão: RN com Ig } 24 \text { a } 32 \text { sema- } \\
\text { nas e peso de nascimento entre } 500 \mathrm{~g} \\
\text { e } 1250 \mathrm{~g} . \\
\text { Exclusão: RN com anomalia congê- } \\
\text { nita do trato gastrintestinal; } \\
\text { enterocolite necrosante; grandes ci- } \\
\text { rurgias, aleitamento materno antes do } \\
\text { início do estudo; paciente com } \\
\text { malformação incompatível com a vida. } \\
\text { Exclusão durante estudo: Sepse } \\
\text { com interrupção da dieta, cirurgia e } \\
\text { óbito. } \\
\text { Período: Março } 1992 \text { a julho } 1994\end{array}$ & $\begin{array}{l}\text { RN divididos em } 2 \text { grupos: } 35 \text { trata- } \\
\text { dos e } 33 \text { controles. } \\
\text { Glutamina: acrescentada na fórmula } \\
\text { láctea } 0,08 \mathrm{~g} / \mathrm{kg} \text { no início ( } 3^{\circ} \mathrm{dia} \text { ), sen- } \\
\text { do aumentado } 0,31 \mathrm{~g} / \mathrm{kg} \text { por dia até } \\
13^{\circ} \text { dia. A glutamina foi suspensa no } \\
30^{\circ} \text { dia. } \\
\text { Progressão lenta enteral até atingir } \\
\text { nutrição plena em torno de } 150 \mathrm{~mL} / \mathrm{kg} / \mathrm{d} \text {. } \\
\text { Suspensa a NP se infusão inferior a } \\
1,5 \mathrm{~mL} / \text { hora. } \\
\text { Controles: dosagem de AA, eletrólitos, } \\
\text { gasometria, amônia. } \\
\text { Todos os RN foram avaliados nos pri- } \\
\text { meiros } 30 \text { dias de vida. }\end{array}$ & $\begin{array}{l}\text { RN suplementados com glutamina: } \\
\text { - Redução dos custos devido à di- } \\
\text { minuição da incidência de sepse; } \\
\text { menor tempo de NP, menor tem- } \\
\text { po de UTI e de VPM e menos } \\
\text { exames laboratoriais. } \\
\text { - Não houve diferença significante } \\
\text { quanto ao tempo de internação } \\
\text { hospitalar. }\end{array}$ \\
\hline
\end{tabular}

$\mathrm{RN}=$ recém-nascidos; Ig=idade gestacional; $\mathrm{AA}=$ aminoácido; $\mathrm{NP}=$ nutrição parenteral; $\mathrm{UTI}=$ unidade de terapia intensiva; VPM= ventilação pulmonar mecânica.

Quadro 4. Efeitos da suplementação oral de glutamina sobre a gravidade da estomatite pós-quimioterapia citotóxica.

\begin{tabular}{|c|c|c|c|}
\hline Autor & Participantes & Intervenção & Resultados \\
\hline $\begin{array}{l}\text { Anderson } \\
\text { et al. }{ }^{9}\end{array}$ & $\begin{array}{l}193 \text { pacientes pediátricos e adultos } \\
\text { submetidos a TMO (87 autólogo; } 55 \\
\text { heterólogo de doador relacionado; e } \\
51 \text { de doador não relacionado). } \\
\text { Indicação de TMO: LLA, LMC, LLNA, } \\
\text { SMD, Tumor sólido, anemia aplásica, } \\
\text { doença hereditária. } \\
\text { Pacientes submetidos a TMO } \\
\text { autólogo não receberam radioterapia } \\
\text { e quimioterapia (metotrexate) prepa- } \\
\text { ratórias. }\end{array}$ & $\begin{array}{l}\text { Dividiu-se em } 2 \text { grupos para receber } \\
\text { glutamina ou placebo. } \\
\text { Variáveis de prognóstico: gravidade da } \\
\text { mucosite por escalas de dor, capacida- } \\
\text { de para se alimentar, uso de analgési- } \\
\text { co, tempo de NP, infecções e uso de } \\
\text { antibióticos. }\end{array}$ & $\begin{array}{l}\text { - Gravidade e duração da muco- } \\
\text { site, assim como a necessidade } \\
\text { de opióide foi menor nos pacien- } \\
\text { tes com TMO autólogo que usa- } \\
\text { ram glutamina. } \\
\text { - Nos pacientes com TMO hete- } \\
\text { rólogo houve piora da mucosite } \\
\uparrow \text { do uso de opióide nos casos } \\
\text { que receberam enxerto de doa- } \\
\text { dores da própria família. } \\
\text { - Não houve diferença entre } 2 \\
\text { grupos quanto ao uso de anti- } \\
\text { bióticos, NP, infecções bacte- } \\
\text { rianas e fúngicas, e GVHD aguda } \\
\text { e crônica. } \\
\text { - A mortalidade precoce foi me- } \\
\text { nor no grupo que usou gluta- } \\
\text { mina, mas essa diferença não se } \\
\text { manteve a longo prazo. }\end{array}$ \\
\hline
\end{tabular}

$\mathrm{TMO}=$ transplante de medula óssea; LLA= leucemia linfoblástica aguda; LMC= leucemia mielóide crônica; LLNA= leucemia não linfocítica aguda; $\mathrm{SMD}=$ síndrome mielodisplásica; $\mathrm{GVHD}=$ doença do hospedeiro contra o enxerto. 
Quadro 5. Efeitos da suplementação de glutamina via enteral em crianças gravemente doentes (estudo piloto).

\begin{tabular}{|c|c|c|c|}
\hline Autor & Participantes & Intervenção & Resultados \\
\hline $\begin{array}{l}\text { Barbosa } \\
\text { et al. }{ }^{10}\end{array}$ & $\begin{array}{l}9 \text { pacientes com idade e peso } \\
\text { semelhantes. } \\
\text { Inclusão: idade de } 1 \text { mês a } 2 \text { anos; } \\
\text { tolerando a dieta enteral; albumina } \\
\text { 3,5g/dL, com sepse ou insuficiência } \\
\text { respiratória. } \\
\text { Exclusão: choque; DMOS; AIDS; } \\
\text { câncer; QTX; diabetes; insuficiência } \\
\text { hepática e renal; dieta enteral por } \\
\text { tempo <5 dias. }\end{array}$ & $\begin{array}{l}\text { Grupo de estudo ( } 5 \text { pacientes) recebeu } \\
\text { via sonda enteral, dose } 0,3 \mathrm{~g} / \mathrm{kg} \text { por } 5 \\
\text { dias. } \\
\text { Grupo controle ( } 4 \text { pacientes) recebeu } \\
\text { caseína via sonda enteral por } 5 \text { dias. }\end{array}$ & $\begin{array}{l}\text { - O grupo que recebeu glutamina } \\
\text { apresentou tendência à redução } \\
\text { das complicações infecciosas e } \\
\text { menor mortalidade. } \\
\text { - Não houve diferença quanto à } \\
\text { permanência em UTI e no HC, } \\
\text { tempo de VPM e tolerância à dieta } \\
\text { enteral. }\end{array}$ \\
\hline
\end{tabular}

DMOS= disfunção de múltiplos órgãos e sistemas; QTX= quimioterapia; UTI= unidade de terapia intensiva; VPM= ventilação pulmonar mecânica; $\mathrm{HC}=$ hospital.

Quadro 6. Suplementação enteral de glutamina e morbidade em recém-nascidos com baixo peso ao nascimento.

\begin{tabular}{|c|c|c|c|}
\hline Autor & Participantes & Intervenção & Resultados \\
\hline $\begin{array}{l}\text { Vaughn } \\
\text { et al. }{ }^{14}\end{array}$ & $\begin{array}{l}\text { Selecionados para estudo } 649 \text { recém- } \\
\text {-nascidos. } \\
\text { Inclusão: RN com peso de nascimen- } \\
\text { to 500-1250g e <7 dias de vida. } \\
\text { Exclusão: presença de anomalias } \\
\text { congênitas e cromossômicas; RN com } \\
\text { alta probabilidade de morte dentro } \\
\text { dos } 3 \text { primeiros dias de vida, ECN nos } \\
\text { primeiros } 7 \text { dias de vida. } \\
\text { Exclusão durante estudo : resíduo } \\
>5 \text { mLkg; sangue nas fezes; vômitos; } \\
\text { ECN; transferência de HC e óbito. }\end{array}$ & $\begin{array}{l}\text { Pacientes randomizados em } 2 \text { grupos: } \\
314 \text { receberam solução com glutamina } \\
\text { e } 335 \text { receberam placebo. } \\
\text { Placebo: água estéril. } \\
\text { Glutamina: solução com } 0,3 \mathrm{~g} / \mathrm{kg} \text { de } \\
\text { peso de nascimento. } \\
\text { As soluções eram administradas por } \\
\text { sonda enteral } 12 / 12 \mathrm{~h} \text {, diariamente, até } \\
28 \text { dias de vida. }\end{array}$ & $\begin{array}{l}\text { - Excluídos do estudo: } 49 \text { RN gru- } \\
\text { po controle; e } 56 \text { grupo } \\
\text { glutamina. } \\
\text { - Não houve diferença entre os } 2 \\
\text { grupos estudados quanto: IG; } \\
\text { peso de nascimento; uso de } \\
\text { corticóide neonatal; RIO; uso ma- } \\
\text { terno de ATB; tipo de parto; sexo; } \\
\text { raça; e Apgar. } \\
\text { - Suplementação com glutamina } \\
\text { não reduziu a incidência de: sepse } \\
\text { nosocomial, de ECN e de morta- } \\
\text { lidade. } \\
\text { - Não houve diferença quanto cres- } \\
\text { cimento; e níveis séricos de } \\
\text { creatinina e albumina. } \\
\text { - Suplementação com glutamina } \\
\text { mostrou tendência a aumentar a } \\
\text { tolerância à dieta, assim como di- } \\
\text { minuir as seqüelas neurológicas } \\
\text { graves. }\end{array}$ \\
\hline
\end{tabular}

$\mathrm{ECN}=$ enterocolite necrosante; $\mathrm{HC}=$ hospital; $\mathrm{IG}=$ idade gestacional; $\mathrm{RIO}=$ risco de infecção ovular; $\mathrm{ATB}=$ antibióticos; $\mathrm{RN}=$ recém-nascidos . 
Quadro 7. Estudo controlado e randômico sobre o uso parenteral de glutamina em recém-nascidos com baixo peso ao nascimento.

\begin{tabular}{|c|c|c|c|}
\hline Autor & Participantes & Intervenção & Resultados \\
\hline $\begin{array}{l}\text { Thompson } \\
\text { et al. } .^{15}\end{array}$ & $\begin{array}{l}\text { Selecionados para estudo } 35 \text { RN } \\
\text { Inclusão: RN com peso de nascimen- } \\
\text { to <1500g, em uso NP. } \\
\text { Exclusão: presença de anomalias } \\
\text { congênitas do TGl, insuficiência he- } \\
\text { pática ou renal e malformações incom- } \\
\text { patíveis com a vida. } \\
\text { Exclusão durante estudo: Uso de } \\
\text { NP por <7 dias. }\end{array}$ & $\begin{array}{l}\text { Pacientes randomizados em } 2 \text { grupos: } \\
17 \text { receberam suplementação EV de } \\
\text { glutamina e } 18 \text { foram controles. } \\
\text { Solução de glutamina } 2,5 \% \text { infunsão } \\
\text { paralela a NP. A glutamina representa- } \\
\text { va } 16 \% \text { do total de AA. } \\
\text { A nutrição enteral nos } 2 \text { grupos com } \\
\text { início no } 3^{\circ} \text { dia, por SOG. } \\
\text { NP início } 1^{\circ} \text { dia / suspensão quando } \\
\text { dieta enteral representava } 80 \% \text { da } \\
\text { oferta. } \\
\text { Controles diários: pH, uréia, leucócitos } \\
\text { e eletrólitos; semanais: dosagem de } \\
\text { amônia, AA, lactato e função hepática. } \\
\text { Controle clínico de sinais de intolerân- } \\
\text { cia a dieta. }\end{array}$ & $\begin{array}{l}\text { - Não houve diferença entre os } 2 \\
\text { grupos estudados quanto: IG; } \\
\text { peso de nascimento; sexo e CRIB. } \\
\text { - A glutamina foi segura e } \\
\text { apresentou boa tolerância, não } \\
\text { havendo diferença nos níveis de } \\
\text { AA, glutamina e glutamato nos } \\
2 \text { grupos estudados. } \\
\text { - Suplementação com glutamina } \\
\text { não afetou a incidência de sepse } \\
\text { nosocomial, leucopenia e ganho } \\
\text { de peso. } \\
\text { - A suplementação com glutamina } \\
\text { reduziu tempo para se atingir } \\
\text { nutrição enteral plena, com } \\
\text { diminuição dos dias de NP. }\end{array}$ \\
\hline
\end{tabular}

$\mathrm{RN}=$ recém-nascidos; $\mathrm{NP}=$ nutrição parenteral; $\mathrm{TGI}=$ trato gastrintestinal; $\mathrm{EV}=$ endovenoso; $\mathrm{AA}=$ aminoácidos; SOG= sonda orogástrica; $\mathrm{IG}=$ idade gestacional; $C \mathrm{CRIB}=$ escore clínico de risco para recém-nascidos.

Apesar das pesquisas sobre os benefícios da glutamina, seu uso ainda não é rotineiro como recurso terapêutico-nutricional, em unidades de cuidados intensivos pediátricos e neonatais. Com base em estudos controlados, alguns questionamentos a respeito do uso deste aminoácido em pacientes pediátricos fundamentaram esta revisão.

Dietas parenterais e enterais com glutamina podem ter resultados benéficos ao prematuro, devido às características intrínsecas deste aminoácido, precursor de purina e pirimidina; quais sejam: promover o crescimento da criança; favorecer a maturação do trato gastrintestinal; prevenir a enterocolite necrosante; melhorar o balanço nitrogenado através da redução do catabolismo protéico; proporcionar melhor desenvolvimento e funcionalidade das células do sistema imune, possibilitando maior resistência às infecções ${ }^{13,19,20}$.

A suplementação enteral e parenteral da glutamina mostrou-se segura em pacientes pediátricos. O uso deste aminoácido não ocasionou níveis tóxicos de amônia e glutamato, nem se mostraram alterações sugestivas de neurotoxici-

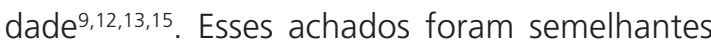
àqueles encontrados em 1990, em estudos feitos em adultos por Ziegler et al. ${ }^{18}$ e confirmados em recente estudo ${ }^{21}$ randômico controlado com recém-nascidos.

Nos estudos neonatais com a suplementação da glutamina, observaram-se a melhor aceitação da dieta enteral e a conseqüente redução do tempo de nutrição parenteral ${ }^{11-15}$. No entanto, a heterogeneidade dos grupos de recém-nascidos estudados limita a interpretação clínica destes resultados.

Em relação aos lactentes e às crianças maiores, não há trabalhos, até o momento, que demonstrem benefícios da glutamina em promover melhor tolerância à dieta enteral9,10. O assunto também continua controverso em relação aos adultos, visto que os estudos, até o momento com pacientes pós-transplante de medula óssea e aqueles criticamente enfermos, demonstram 
apenas tendências a efeitos benéficos, que não chegam a ser significativos ${ }^{17,22}$.

Gianotti et al., em 199523, estudaram a atuação da glutamina na mucosa intestinal, observando que esta evitava a translocação bacteriana - definida como a passagem de microorganismos e suas toxinas do intestino para os órgãos sistêmicos, levando à sepse e à possível evolução para síndrome da disfunção de múltiplos órgãos. Estes autores verificaram que, os camundongos alimentados com dietas enriquecidas com glutamina, apresentaram menor grau de translocação e maior sobrevivência que o grupo controle.

Muitos estudos em humanos foram desenvolvidos nos últimos anos, tendo em vista a possibilidade da glutamina diminuir as infecções, tanto por sua ação na mucosa intestinal, como por sua atuação direta sobre as células do sistema imune ${ }^{24}$, as quais utilizam a glutamina como substrato para obter energia e precursores para biossíntese de citocinas. Grande parte destas pesquisas foram realizadas com adultos gravemente enfermos, pós-transplante de medula óssea, queimados e vítimas de politraumas ${ }^{25-27}$. Nestas, verificou-se, dentre outros benefícios, a redução das infecções. Contudo, outros estudos em pacientes pós-transplante de medula óssea, não conseguiram comprovar tais vantagens ${ }^{17,28}$.

Na presente revisão, verificou-se que a redução de infecção foi significante somente nos estudos de Dallas et al. ${ }^{11}$ e Neu et al. ${ }^{13}$, enquanto que no grupo estudado por Barbosa et al. ${ }^{10}$, foi evidenciada apenas uma tendência à redução - fato decorrente, provavelmente, da pequena amostra de pacientes. Nenhuma diferença foi observada nos estudos de Anderson et al. ${ }^{9}$, Lacey et al. ${ }^{12}$, Thompson et al. ${ }^{15}$ e Vaughn et al. ${ }^{14}$. Embora os resultados para a população pediátrica sejam conflitantes, tais divergências também estão presentes nos resultados observados em trabalhos com pacientes adultos ${ }^{8}$, nos quais a redução das infecções foi demonstrada apenas em relação aos pacientes cirúrgicos.
Dois estudos pediátricos abordaram a variável mortalidade; no grupo estudado por Barbosa et al. ${ }^{10}$ houve apenas tendência à redução desta variável, enquanto no grupo de recém-nascidos estudados por Vaughn et al. ${ }^{14}$, a suplementação deste aminoácido não promoveu redução da mortalidade. Esses dados são compatíveis com os resultados encontrados em estudos randômicos e controlados, realizados em adultos internados em UTI, queimados e em pós-operatório. Nestes estudos, a suplementação da glutamina não promoveu redução da mortalidade em pacientes pós-cirúrgicos, mostrando apenas tendência à redução desta variável em pacientes criticamente enfermos $8,25,29-32$.

Apesar da presente revisão não evidenciar vantagens do uso da glutamina em reduzir o tempo de internação $0^{10,11,13}$, sendo detectada apenas uma tendência a essa redução no estudo de Lacey et al. ${ }^{12}$, a literatura evidencia reduções significantes no tempo de hospitalização, nos estudos com adultos pós-cirúrgicos, com doenças hematológicas e com adultos criticamente enfermos $8,22,27,28,31,32$

Há um grande questionamento quanto ao uso da glutamina ser ou não economicamente viável, ou seja, busca-se saber se os benefícios alcançados com a suplementação superam os investimentos $^{33}$. É sabido que a administração da glutamina, seja enteral ou parenteral, aumenta os gastos com nutrição; contudo, estudos randômicos e controlados em adultos ${ }^{31}$, têm demonstrado que o uso da glutamina é capaz de promover redução do custo hospitalar, à medida que, devido a esse uso, diminuem os gastos com nutrição parenteral, exames laboratoriais e tempo de internação. Entretanto, como ocorre com os estudos pediátricos, somente um ensaio abordou este assunto, revelando redução dos custos ${ }^{11}$. Portanto, torna-se difícil, e até precipitado, chegar-se a alguma conclusão sobre as eventuais vantagens econômicas da suplementação de glutamina em crianças.

Pela análise dos estudos da literatura, não há dados convincentes para que se adote o uso 
rotineiro da glutamina em pacientes pediátricos criticamente enfermos. Os poucos estudos controlados e randômicos nesta faixa etária, não permitem comparações, por terem utilizado amostras heterogêneas e administrado a glutamina em doses diversas e por vias distintas. Nesse contexto, entretanto, foram observados benefícios tais como a redução no tempo de uso da nutrição parenteral, maior tolerância à nutrição enteral (principalmente em recém-nascidos) e uma tendência à redução de infecções hospitalares ${ }^{34,35}$. Portanto, a suplementação de glutamina em crianças criticamente doentes, permanece um campo aberto a novas pesquisas clínicas.

\section{REFERÊ NCIAS}

1. Hall JC, Heel K, McCauley R. Glutamine. Br J Surg. 1996; 83(3):305-12.

2. Lacey JM, Wilmore DW. Is glutamine conditionally essential amino acid? Nutr Rev. 1990; 48(8): 297-309.

3. Muhlbacher F, Kapadia CR, Colpoys MF, Smithth WDW. Effects of glucocorticoids on glutamine metabolism in skeletal muscle. Am J Physiol. 1984; 247(1):75-83.

4. Murphy C, Newsolme P. Macrophage mediated lysis of a beta-cell line, tumor necrosis factor-alpha release from bacillus Calmetti-Guerin (BCG) activated murine macrophages and interleukin-8 release from human monocytes are dependent on extracellular glutamine concentration and glutamine metabolism. Clin Sci. 1999; 96(1): 89-97.

5. Wilmore DW, Shabert JK. Role of glutamine in immunologic responses. Nutrition. 1998; 14 (7-8):618-26.

6. Alverdy JC Effects of glutamine suplemented diets on imunology of the gut. JPEN J Parenter Enteral Nutr. 1990; 14(4):109S-13S.

7. Kelly D, Wischmeyer PE. Role of L-glutamine in critical illness: new insights. Curr Opin Clin Nutr Metab Care. 2003; 6(2):217-22.
8. Novak F, Heyland DK, Avenell A, Drover JW, Su X. Glutamine supplementation in serious illness: a systematic review of the evidence. Crit Care Med. 2002; 30(9):2022-9.

9. Anderson PM, Schroeder G, Skubitz KM. Oral glutamine reduces the duration and severity of stomatitis after cytotoxic cancer chemotherapy. Cancer. 1998; 83(7):1433-9.

10. Barbosa E, Moreira EA, Goes JE, Faintuch J. Pilot study with a glutamine-supplemented enteral formula in critically ill infants. Rev Hosp Clin Fac Med São Paulo. 1999; 54(1):21-4.

11. Dallas MJ, Bowling D, Roig JC, Auestad N, Neu J. Enteral glutamine supplementation for very-low-birth-weight infants decreases hospital costs. J Parenter Enteral Nutr 1998; 22(6):352-6.

12. Lacey JM, Crouch JB, Benfell K, Ringer SA, Wilmore $C K$, Maguire $D$, et al. The effects of glutaminesupplemented parenteral nutrition in premature infants. J Parenter Enteral Nutr. 1996; 20:74-80.

13. Neu J, Roig JC, Meetze WH, Veerman M, Carter C, Millsaps $M$, et al. Enteral glutamine supplementation for very low birth weight infants decreases morbidity. J Pediatr. 1997; 131:691-9.

14. Vaughn P, Thomas P, Clark R, Neu J. Enteral glutamine supplementation and morbidity in low birth weight infants. J Pediatr. 2003; 142(6): 662-8.

15. Thompson SW, McClure BG, Tubman TRJ. A randomized, controlled trial of parenteral glutamine in ill, very low birth-weight neonates. J Pediatr Gastroenterol Nutr. 2003; 37(5):550-3.

16. Houdijk AP, Nijveldt RJ, van Leeuwen PA. Glutamine-enriched enteral feeding in trauma patients: reduced infectious morbidity is not related to changes in endocrine and metabolic responses. J Parenter Enteral Nutr. 1999; 23(5): 52S-8S.

17. Schloerb PR, Skikne BS. Oral and parenteral glutamine in bone marrow transplantation: a randomized, double-blind study. J Parenter Enteral Nutr 1999; 23(3):117-22.

18. Ziegler TR, Benfell K, Smith RJ, Young LS, Brown E, Ferrari-Baliviera $\mathrm{E}$, et al. Safety and metabolic effects 
of glutamine administration in humans. J Parenter Enteral Nutr. 1990; 14:137S-46S.

19. Neu J, DeMarco V, Li N. Glutamine: clinical applications and mechanisms of action. Curr Opin Clin Nutr Metab Care. 2002; 5(1):69-75.

20. Tubman TR, Thompson SW. Glutamine supplementation for prevention of morbidity in preterm infants. Cochrane Database Syst Rev. 2001; (4):CD001457.

21. Poindexter BB, Ehrenkranz RA, Stoll BJ, Koch MA,

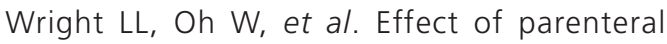
glutamine supplementation on plasma amino acid concentrations in extremely low-birth-weight infants. Am J Clin Nutr. 2003; 77(3):737-4

22. Coghlin Dickson TM, Wong RM, Offrin RS, Shizuru JA, Johnston LJ, Hu WW, et al. Effect of oral glutamine supplementation during bone marrow transplantation. J Parenter Enteral Nutr. 2000; 24(2):61-6.

23. Gianotti L, Alexander JW, Gennari R, Pyles T, Babcock GF. Oral glutamine decreases bacterial translocation and improves survival in experimental gut-origin sepsis. J Parenter Enteral Nutr. 1995; 19(1):69-74

24. Karinch AM, Pan M, Lin CM, Strange $R$, Souba WW. Glutamine metabolism in sepsis and infection. J Nutr. 2001; 131(9):2535S-8S.

25. Wischmeyer PE, Lynch J, Liedel J, Wolfson R, Riehm J, Gottlieb L, et al. Glutamine administration reduces Gram-negative bacteremia in severely burned patients: a prospective, randomized, double-blind trial versus isonitrogenous control. Crit Care Med. 2001; 29(11):2075-80.

26. Houdijk AP, Rijnsburger ER, Jansen J, Wesdorp RI, Weiss JK, McCamish MA, et al. Randomised trial of glutamine-enriched enteral nutrition on infectious morbidity in patients with multiple traumas. Lancet. 1998; 352(9130):772-6.
27. Garcia-de-Lorenzo A, Zarazaga A, Garcia-Luna PP, Gonzalez-huiz F, Lopez-Martinez J, Mijan A. Clinical evidence for enteral nutritional support with glutamine: a systematic review. Nutrition. 2003; 19(9):805-11.

28. Schloerb PR, Amare M. Total parenteral nutrition with glutamine in bone marrow transplantation and other clinical applications. J Parenter Enteral Nutr. 1993; 17(5):407-13.

29. Goeters C, Wenn A, Mertes N, Wempe C, Van Aken $\mathrm{H}$, Stehle $\mathrm{P}$, et al. Parenteral L-alanyl-L-glutamimine improves 6 month outcome in critically ill patients. Crit Care Med. 2002; 30(9):2032-7.

30. Griffiths RD, Allen KD, Andrews FJ, Jones C. Infection, multiple organ failure, and survival in the intensive care unit: influence of glutamine-supplemented parenteral nutrition on acquired infection. Nutrition. 2002; 18(7-8):546-52.

31. Mertes N, Schulzki C, Goeters C, Winde G, Benzing $\mathrm{S}$, Keihn KS. et al. Cost containment through L-alanyl-L-glutamine supplemented total parenteral nutrition after major abdominal surgery: a prospective randomized double-blind controlled study. Clin Nutr. 2000; 19(6):395-401.

32. Powell-Tuck J. Total parenteral nutrition with glutamine dipeptide shortened hospital stays and improved immune status and nitrogen economy after major abdominal surgery. Gut. 1999; 44(2):155

33. Ziegler TR, Bazargan N, Galloway JR. Glutamine supplemented nutrition suport: saving nitrogen and saving money? Clin Nutr. 2000; 19(6):375-7.

34. Ball PA, Hardy G. Glutamine in pediatrics: where next? Nutrition. 2002; 18(6):451-4.

35. Neu J. Glutamine supplements in premature infants: why and how. J Pediatr Gastroenterol Nutr. 2003; 37(5):533-5

Recebido para publicação em 19 de setembro de 2003 e aceito em 17 de maio de 2004 